11

\title{
K теории инициирующего действия непрерывного нагрева на структурные перестройки и температуру фазового перехода в кристаллах
}

\author{
(C) Ю.А. ХоH \\ Институт физики прочности и материаловедения СО РАН, \\ Томск, Россия \\ E-mail: khon@ispms.ru
}

Поступила в Редакцию 19 декабря 2019 г.

В окончательной редакции 19 декабря 2019 г.

Принята к публикации 21 декабря 2019 г.

\begin{abstract}
Предложены модель и кинетическая теория структурных изменений в кристаллах при непрерывном нагреве, учитывающая неадиабатические переходы атомов между различными состояниями системы ядер и электронов. Неадиабатические переходы инициируют структурные перестройки, определяемые изменением ближнего порядка при перераспределении электронной плотности. Показано, что непрерывный нагрев стимулирует структурные изменения в кристалле и понижает температуру известного фазового перехода.
\end{abstract}

Ключевые слова: фазовый переход, смешанное состояние, динамические параметры порядка, температура фазового перехода.

DOI: 10.21883/FTT.2020.06.49355.659

\section{1. Введение}

Классическая теория равновесных фазовых переходов первого рода основана на модели зарождения и роста [1-4]. Новая фаза начинает расти, если зародыш новой фазы превышает критический размер. Предполагается, что образование закритического зародыша определяется флуктуациями при тепловых колебаниях атомов. Как следствие, при увеличении скорости нагрева время образования закритического зародыша должно уменьшаться, а температура фазового перехода первого рода повышаться. Однако этот вывод противоречит имеющимся экспериментальным данным, полученным в [5] на кристаллах прустита $\left(\mathrm{Ag}_{3} \mathrm{AsS}_{3}\right)$. В этой системе в равновесных условиях фазовый переход первого рода происходит при температуре $T_{c 0}=28-30 \mathrm{~K}$. Установлено, что при нагреве с постоянной скоростью температура неравновесного фазового превращения $T_{c}$ понижается. Разность температур $\Delta T=T_{c}-T_{c 0}$ зависит от скорости нагрева $\dot{T}$. При увеличении $\dot{T}$ на кривой зависимости $\Delta T=\Delta T(\dot{T})$ имеются две стадии. На первой стадии $\Delta T$ уменьшается, а затем при $\dot{T}>(8-10) \mathrm{K} / \mathrm{min}$ возрастает. В точке минимума $\Delta T \approx 3 \mathrm{~K}$. Примечательно, что неравновесному фазовому превращению предшествует возбуждение метастабильного ближнего порядка, время жизни которого при постоянной температуре оценивается в $\approx 16 \mathrm{~min}$. Происхождение данного ближнего порядка и его влияние на температуру фазового перехода до сих пор остается загадочным.

Возможная причина такого положения дел состоит в следующем. В рамках классической теории зарождения и роста неявно предполагается, что смещения атомов в системе атомов при ее нагреве полностью определяются термическими флуктуациями. Строго говоря, это приближение справедливо для изолированной системы вблизи положения равновесия. С точки зрения квантовой механики такие системы находятся в чистом состоянии, динамика атомов является адиабатической. На самом деле при нагреве, а тем более, при нагреве с конечной скоростью среда представляет открытую неравновесную систему, находящуюся в смешанном состоянии. Смешанное состояние представляет некогерентную смесь чистых состояний. Во внимание должны приниматься не только колебательные, но и электронные степени свободы. Динамика атомов становится неадиабатической [6,7]. Последовательное решение задачи о структурных изменениях в кристалле на расстояниях десятков-тысяч нанометров и диффузионных временах в рамках неадиабатической молекулярной динамики вряд ли возможно. Требуются новые подходы и модели. В настоящей работе предлагается модель, учитывающая возбуждение динамического ближнего порядка в смешанном состоянии системы и его влияние на температуру фазового перехода первого рода при непрерывном нагреве.

\section{2. Модель структурных изменений в кристалле при непрерывном нагреве}

Рассматривается кристалл, в котором при фазовом переходе первого рода меняется только топологический ближний порядок. При температуре $T<T_{c}$ свободная энергия первой фазы $F_{1}\left(T, V_{1}\right)$ меньше свободной энергии $F_{2}\left(T, V_{2}\right)$ второй фазы. Здесь $V_{1}, V_{2}$ - объемы 
фаз 1, 2 соответственно. При температуре фазового перехода $F_{1}\left(T_{c}, V_{1}\right)=F_{2}\left(T_{c}, V_{2}\right)$. Объем системы меняется от $V_{1}$ до $V_{2}$. При $T>T_{c} F_{1}>F_{2}$.

При нагреве кристалла система ядер и электронов удаляется от состояния равновесия и находится в смешанном состоянии в виде некогерентной смеси чистых состояний со статистическими весами $c_{i}$. Здесь $i-$ номер чистого состояния. Статистические веса - вещественные положительные числа, удовлетворяющие условию $\Sigma_{i} c_{i}=1$. В дальнейшем индексами $i=1,2$ будут обозначаться чистые состояния фаз 1, 2 соответственно. Каждое чистое состояние характеризуется своим распределением ядер и электронов, ближним порядком $\xi_{i}$, химической связью между атомами. Потенциальная энергия системы в смешанном состоянии $U(R)$ представляет совокупность пересекающихся гиперповерхностей (ГП) потенциальных энергий $U_{i}(R)$ чистых состояний. Здесь $R$ - совокупность координат атомов. Естественно, что проявляются те чистые состояния, при возбуждении которых потенциальная энергия системы уменьшается. Динамика атомов в смешанном состоянии системы определяется движением по выделенной ГП и переходами с этой ГП на другую [6,7]. Имеются два пути перехода. Первый путь определяется переходами через потенциальный барьер при тепловых флуктуациях. Характерная частота перехода определяется высотой потенциального барьера и температурой. Второй путь определяется неадиабатическими переходами Ландау-Зинера вблизи линий пересечения ГП $[8,9]$. Характерная частота таких переходов не зависит от температуры и определяется изменением химических связей при перераспределении электронной плотности.

Решение уравнений неадиабатической молекулярной динамики позволяет, в принципе, найти координаты атомов, число чистых состояний, ближний порядок, потенциальную энергию в любой момент времени. В классическом подходе предполагается, что система, первоначально находящаяся на ГП $U_{1}$, остается на ней $\left(c_{1}=1\right)$ вплоть до температуры фазового перехода. Переход на ГП $U_{2}$ происходит по первому пути. Атомы должны сместиться на величину $u_{21}=R_{2}-R_{1}$, здесь $R_{2}, R_{1}$ - координаты выделенного атома во второй и первой фазах соответственно. Сам факт обнаружения указанного выше метастабильного ближнего порядка означает, что при удалении от состояния равновесия происходит переход на ГП $U_{3}$, и возбуждается ближний порядок $\xi_{3}$, нехарактерный для фазы 1. Система находится в смешанном состоянии со статистическими весами $c_{1}>0, c_{3}>0$. Возбуждение ближнего порядка $\xi_{3}$ определяется изменением химической связи. Ближний порядок такого типа был назван динамическим ближним порядком [10,11]. Переход на ГП $U_{3}<U_{1}$ определяется смещениями $u_{31}=R_{3}-R_{1}, R_{3}-$ координаты атомов в кластерах динамического ближнего порядка. В смешанном состоянии твердое тело представляет смесь кластеров атомов с ближними порядками $\xi_{1}, \xi_{3}$. Кластеры с ближним порядком $\xi_{3}$ можно рассматривать в качестве докритических зародышей. Переход на ГП $U_{2}<U_{3}$ определяется смещениями атомов $u_{23}=R_{2}-R_{3}$. Смещение $u_{23}$ может быть достаточно малым, чтобы переход на ГП $U_{2}$ происходил за счет тепловых флуктуаций с малой амплитудой. В дальнейшем предполагается, что это условие выполняется. Динамика системы на больших пространственных $(r)$ и временных $(t)$ масштабах определяется числом атомов $N_{i}(r, t)$ на ГП $U_{i}$. При этом $\Sigma_{i} N_{i}=N$ (здесь $N$ - общее число атомов). Введем две переменные $\eta=N_{2} / N, \varphi=N_{3} / N$. В фазе 1 $\eta=0, \varphi=0$, в фазе $2 \eta=1, \varphi=0$. В дальнейшем для определенности переменные $0 \leq \eta \leq 1,0 \leq \varphi \leq 1$ будем называть динамическими параметрами порядка (ДПП). Это название подчеркивает, что ДПП порядка описывают состояние неравновесной открытой системы. В классическом подходе рассматривается только один ДПП $\eta$. В рассматриваемой модели наблюдаемый экспериментально ближний порядок, который возбуждается при непрерывном нагреве [5], ассоциируется с ДПП $\varphi$.

Кинетические уравнения для ДПП не могут быть получены вариацией термодинамического потенциала. Тем не менее, качественный вид этих уравнений может быть получен, исходя из следующих соображений. Изменение ДПП может рассматриваться как результат химических реакций, в которых ближний порядок одного типа превращается в ближний порядок другого типа. Тогда ДПП формально представляют концентрации кластеров с характерным для них ближним порядком. В рамках реакционно-диффузионной модели безразмерные уравнения эволюции могут быть записаны в виде

$$
\begin{gathered}
t_{\eta} \frac{\partial \eta}{\partial t}=H_{0}(\eta)+H_{1}(\eta, \varphi)+l_{\eta}^{2} \Delta \eta, \\
t_{\varphi} \frac{\partial \varphi}{\partial t}=\Phi_{0}(\varphi)+\Phi_{1}(\eta, \varphi)+l_{\varphi}^{2} \Delta \varphi .
\end{gathered}
$$

Здесь $H_{0}, H_{1}, \Phi_{0}, \Phi_{1}$ называются функциями источников, функции $H_{1}(\eta, \varphi=0)=0, \Phi_{1}(\eta=0, \varphi)=0, t_{\eta}, t_{\varphi}, l_{\eta}$, $l_{\varphi}$ имеют смысл характерного времени и характерной длины изменения переменных $\eta, \varphi$ соответственно. По порядку величины параметр $l_{\eta}$ равен нескольким межатомным расстояниям, $l_{\varphi}$ - доле межатомным расстояний.

Уравнение (1) при $H_{1}=0$ должно иметь два однородных стационарных решения $\eta=0$ и $\eta=1$. Каждое решение может быть абсолютно устойчивым, относительно устойчивым и неустойчивым. Оба состояния имеют одинаковую устойчивость при температуре фазового перехода. Такой тип устойчивости решений характерен для бистабильной среды. Простейшей функцией, описывающей такую среду, является функция

$$
H_{0}=-\gamma \eta\left(\eta-\eta_{1}\right)(\eta-1) .
$$

Здесь параметр $\eta_{1}=\eta_{1}(T)$ определяет устойчивость состояний, параметр $\gamma$ - высоту потенциального барьера, разделяющего два устойчивых состояния. Оба состояния $\eta=0, \eta=1$ имеют одинаковую устойчивость при 
$\eta_{1}=1 / 2$. Состояние $\eta=0$ стабильно (метастабильно), а состояние $\eta=1$ метастабильно (стабильно) при $\eta_{1}>\frac{1}{2}$ $\left(1 / 2<\eta_{1}\right)$. Уравнение $\eta_{1}\left(T_{c}\right)=\frac{1}{2}$ определяет температуру равновесия фаз. В линейном по параметру $\eta$ приближении функция $H_{1}$ имеет вид

$$
H_{1}(\eta, \varphi)=d_{0} \varphi \eta
$$

Коэффициент связи $d_{0}>0$ считается постоянным.

Учитывая, что динамический ближний порядок может быть метастабильным, функция $\Phi_{0}$ записана в виде

$$
\Phi_{0}=\alpha \varphi+\beta \varphi^{2}-\varphi^{3} .
$$

Здесь параметры $\alpha, \beta$ определяют величину и устойчивость решений $\varphi_{0}=\varphi=0$ и

$$
\varphi_{s}=\frac{\beta}{2}+\left(\frac{\beta^{2}}{4}+\alpha\right)^{1 / 2} .
$$

Величина $\varphi_{s}<1$ при $\beta<1-\alpha$. Решение $\varphi_{0}$ является единственным и устойчивым при $\alpha<-\beta^{2} / 4$. При $\alpha>0$ решение $\varphi_{s}$ абсолютно устойчиво, решение $\varphi_{0}$ неустойчиво. При $-\beta^{2} / 4<\alpha<-2 \beta^{2} / 9$ решение $\varphi_{0}$ абсолютно устойчиво, $\varphi_{s}$ относительно устойчиво (метастабильно). При $-2 \beta^{2} / 9<\alpha<0$ решение $\varphi_{0}$ метастабильно, решение $\varphi_{s}$ абсолютно устойчиво. Параметр $\alpha$ определяется скоростями процессов возбуждения и распада кластеров динамического ближнего порядка. При $\alpha>-2 \beta^{2} / 9$ процессы возбуждения доминируют над процессами распада. Процессы распада определяются тепловыми колебаниями атомов, а процессы возбуждения скоростью изменения состояния среды, в рассматриваемом случае скоростью нагрева. Поэтому параметр $\alpha$ является возрастающей функцией скорости нагрева.

В линейном по $\varphi$ приближении функция источника $\Phi_{1}$ имеет вид

$$
\Phi_{1}(\eta, \varphi)=-c \varphi \eta
$$

Положительный знак коэффициента связи $c$ означает, что образование новой фазы при фазовом переходе уменьшает скорость возрастания $\varphi$. В равновесных состояниях $(\eta=0, \eta=1)$ уравнение (2) должно иметь единственное однородное решение $\varphi=0$, что имеет место при $c \geq \alpha$.

С учетом (3)-(5),(7) уравнения (1),(2) принимают вид

$$
\begin{gathered}
t_{\eta} \frac{\partial \eta}{\partial t}=-\eta_{1} \eta+\left(1+\eta_{1}\right) \eta^{2}-\eta^{3}+d \varphi \eta+l_{\eta}^{2} \Delta \eta . \\
t_{\varphi} \frac{\partial \varphi}{\partial t}=(\alpha-c \eta) \varphi+\beta \varphi^{2}-\varphi^{3}+l_{\varphi}^{2} \Delta \varphi .
\end{gathered}
$$

Коэффициент $\gamma$ в правой части (3) включен в $t_{\eta}$ и $l_{\eta}$, коэффициент $d=d_{0} / \gamma$. Система связанных уравнений (8), (9) описывает динамику изменения динамических параметров порядка.

\section{3. Влияние скорости нагрева на температуру фазового перехода}

Уравнения (8), (9) имеют три однородных стационарных решения: 1) $\eta=0, \varphi=0$; 2) $\eta=1, \varphi=0$; 3) $\eta=0$, $\varphi=\varphi_{s}$. Первое (второе) решение описывает устойчивую фазу 1 (2), а третье - фазу 1 с возбужденным динамическим ближним порядком. Стандартный линейный анализ устойчивости однородных решений показывает, что решение $\eta=0, \varphi=0$ неустойчиво относительно малых возмущений с амплитудой $\delta \varphi$ при

$$
\frac{t_{\varphi}}{t_{\eta}}<\alpha / \eta_{1}
$$

Это неравенство выполняется, если $\alpha>0$ и $t_{\varphi} \ll t_{\eta}$. Второе неравенство дает возможность рассматривать стационарные значения $\varphi_{s}$.

Рассмотрим вначале фазовый переход из состояния $\eta=0$ в состояние $\eta=1$ в равновесных условиях (при малых скоростях нагрева). Обозначим через $\alpha_{0}$ значения параметра $\alpha$ в (9) при равновесном фазовом переходе. Тогда стационарное значение переменой $\varphi$ будет равно $\varphi_{s 0}=\frac{\beta}{2}+\left(\frac{\beta^{2}}{4}+\alpha_{0}\right)^{1 / 2}$. При гетерогенном механизме фазового перехода в равновесных условиях решение $\eta=0, \varphi=\varphi_{s}$ должно быть устойчиво относительно малых однородных и неоднородных возмущений $\delta \eta$, но неустойчиво относительно возмущений с амплитудой $\Delta \eta>\Delta \eta_{c}$. Из (8), (9) следует, что

$$
\Delta \eta_{c}=\eta_{1}-d \varphi_{s 0} .
$$

В качестве возмущений с амплитудой $\Delta \eta_{c}$ выступают имеющиеся в системе, либо образующиеся при тепловых флуктуациях зародыши. Как указывалось выше, $\eta_{1}=\eta_{1}(T)$. Из уравнения (11) при фиксированном значении $\Delta \eta_{c}$ может быть найдена температура равновесного фазового перехода $T_{c 0}$. Будем считать ее известной.

В неравновесных условиях параметр $\alpha>\alpha_{0}$, а $\varphi_{s}>\varphi_{s 0}$. Тогда при том же самом значении $\Delta \eta_{c}$ температура неравновесного фазового перехода будет определяться уравнением

$$
\Delta \eta_{c}=\eta_{1}-d \varphi_{s}
$$

При $\varphi_{s}>\varphi_{s 0}$ это уравнение будет иметь решение при $\eta_{1}>1 / 2$. А это означает, что неравновесная температура фазового перехода $T_{c}<T_{c 0}$. При малом изменении $\eta_{1}$ чем больше скорость нагрева, тем больше разность $\Delta T=T_{c 0}-T_{c}$. Это объясняет наблюдаемую экспериментально первую стадию понижения температуры неравновесного фазового перехода при увеличении скорости нагрева. Качественное объяснение второй стадии было дано в [5]. Оно следует из уравнения (12). Температура образца прямо пропорциональна, а параметр $\eta_{1}$ обратно пропорционален скорости нагрева $\dot{T}$. В линейном приближении $\alpha \sim \dot{T}^{1 / 2}$. При высокой скорости нагрева основной вклад в уменьшение правой части (12) 
дает величина $\eta_{1}$. Относительное уменьшение вклада, определяемого вторым слагаемым в правой части (12), по сравнению с первым приводит к уменьшению $\Delta T$ и формированию второй стадии на кривой зависимости $\Delta T$ от $\dot{T}$.

Как указывалось выше при постоянном нагреве параметр $\alpha>\alpha_{0}$, а динамический параметр порядка $\varphi_{s}>\varphi_{s 0}$. Если нагрев прекратить и поддерживать температуру постоянной, то параметр $\alpha$ примет значение, равное $\alpha_{0}$. Система оказывается в неравновесном состоянии. Релаксация к состоянию $\varphi=\varphi_{s 0}$ определяется тепловыми колебаниями атомов с характерным временем $t_{\eta}$. Если принять время релаксации в равновесное состояние $t_{r} \approx 10 t_{\eta}$, то при $t_{r} \approx 10^{3} \mathrm{~s}[5]$ находим $t_{\eta} \approx 10^{2} \mathrm{~s}$. При нагреве с постоянной скоростью интенсивности брэгговских рефлексов в экспериментах по определению времени релаксации метастабильных состояний непрерывно возрастают с увеличением температуры. Это означает, что принятое при анализе условие $t_{\eta} \gg t_{\varphi}$ выполняется. В тех системах, в которых это условие не выполняется, влияние постоянного нагрева на температуру фазового первого рода может быть другим.

\section{4. Заключение}

В предложенной модели влияния непрерывного нагрева на структурные перестройки и температуру фазового перехода первого рода помимо смещений атомов при тепловых колебаниях принято во внимание возбуждение динамического ближнего порядка при неадиабатических переходах между различными состояниями неравновесных открытых систем. Наблюдаемые экспериментально структурные перестройки в кристалле ассоциируются с динамическим ближним порядком. Возбуждение динамического ближнего порядка инициирует неустойчивость системы относительно смещений атомов при тепловых колебаниях и приводит к понижению температуры неравновесного фазового перехода по сравнению с равновесным значением. Развитая модель влияния непрерывного нагрева на температуру фазового перехода первого рода без труда обобщается на случай непрерывного охлаждения системы. В этом случае температура фазового перехода первого рода будет повышаться.

\section{Благодарности}

Авторы благодарят проф. Зуева Л.Б., проф. Слядникова Е.Е. за интерес к работе и полезные замечания.

\section{Финансирование работы}

Работа выполнена в рамках Программы фундаментальных научных исследований государственных академий наук на 2013-2020 годы, направление III.23.

\section{Конфликт интересов}

Авторы заявляют, что у них нет конфликта интересов.

\section{Список литературы}

[1] J.W. Christian. The theory of transformations in metals and alloys. Part I. Equilibrium and general kinetic theory. Pergamon Press (1975).

[2] D.A. Porter, K.E. Easterling. Phase Transformation in Metals and Alloys. Van Nostrand Reinhold, N. Y.(1992).

[3] Ya.B. Zeldovich. J. Exp. Theor. Phys. 12, 525 (1942).

[4] I.M. Lifshitz, V.V. Slyozov. J. Phys. Chem. Solids 19, 35 (1961).

[5] И.М. Шмытько, И.М. Афонникова, Н.С. Дорохова. ФТТ 40, 2217 (1998). [I.M. Shmytko, I.M. Afonnikova, N.S. Dorokhova. Phys. Solid State 40, 2217 (1998)].

[6] R. Kapral. J. Phys.: Condens. Matter 27, 073201 (2015).

[7] J.C. Tully. Int. J. Quantum Chem.: Quantum Chem. Symposium 25, 299 (1991).

[8] L. Landau. Phys. Z. Sowjetunion 2, 46 (1932).

[9] C. Zener. Proc. R. Soc. A 137, 696 (1932).

[10] В.Е. Егорушкин, Н.В. Мельникова. ЖЭТФ 103, 214 (1993). [V.E. Egorushkin, N.V. Mel'nikova. J. Exp. Theor. Phys. 76, 116 (1993)].

[11] M.S. Barabashko, A.E. Rezvanova \& A.N. Ponomarev. Fullerenes, nanotubes carbon nanostructures 25, 11, 661 (2017).

Редактор Т.Н. Василевская 